correlation between number of clinics prior to endoscopy and the time to endoscopy ( $\mathrm{r} 0.83 \mathrm{p}<0.001)$

There were no reported complications.

Conclusions In this study, most OGDs and about half OGD/ Colons are endoscopically and histologically normal. Endoscopic features strongly correlate with histology, questioning the need for routine biopsies and a potential cost saving measure. Negative endoscopies appear to offer reassurance and allowed almost half the children to be discharged from hospital follow up. This is the first study to look at the value of normal endoscopy on clinical outcomes of paediatric patients.

\section{PTU-3 ENDOSCOPIC PALLIATION AND PROGNOSTICATION OF MALIGNANT HILAR BILIARY STRICTURES: A SINGLE CENTRE EXPERIENCE}

Wei On*, Muhammad A Saleem, Vinod S Hegade, Matthew T Huggett, Bharat Paranandi, Simon Everett. Leeds Teaching Hospitals NHS Trust, Leeds, UK

\subsection{6/gutjin-2021-BSG.76}

Introduction The optimum approach to palliation of malignant hilar biliary strictures (MHBS) is unclear. National UK Hospital Episode Statistics data demonstrates 30-day mortality of $>$ $20 \%$ following endoscopic and percutaneous stenting. ${ }^{1,2} \mathrm{We}$ aimed to describe outcomes and predictive factors in patients undergoing ERCP and stenting for MHBS in a single tertiary hepatopancreatobiliary centre.

Methods All patients undergoing index palliative endoscopic stenting for MHBS from February 2015 to September 2020 were identified from a prospective database. Cross-sectional imaging was reviewed and stenting intention planned (left or right unilateral; bilateral) to optimise drainage. All patients received single dose antibiotic prophylaxis. Glasgow Prognostic Score (GPS, a validated tool for prognostication in various malignancies calculated with a 3 point scale based on serum albumin and CRP) was calculated retrospectively.

Results 95 patients (53 male, mean age 70 yrs (range 30-92)) were included. Commonest aetiology was cholangiocarcinoma in $42.7 \%$ followed by metastatic hilar obstruction in $33.7 \%$. Metal stents were placed in $87.4 \%$, plastic in $10.3 \%$, and both in 2.3\%. Stenting intention was achieved in $83.2 \%$ $(84.6 \%$ unilateral intent and $81.4 \%$ bilateral intent). Clinical success (reduction of serum bilirubin to $\leq 50 \%$ its original value within 30 days) was achieved in $84.8 \%$. 30-day adverse event rate was $31.6 \%$, (24.2\% cholangitis). 30-day mortality was $24.2 \%$; mortality stratified by GPS (available in $89 / 95$ ) was: $0 \%$ in GPS0 $(n=4), 27.8 \%$ in GPS1 $(n=18)$ and $25.4 \%$ in GPS2 $(\mathrm{n}=67)$. Neither serum albumin $(<35 \mathrm{~g} / \mathrm{L}$ vs $\geq 35 \mathrm{~g} /$ L; $24.4 \%$ vs $26.7 \%, \mathrm{p}=0.85)$ nor CRP $(<10$ vs $\geq 10 \mathrm{mg} / \mathrm{L}$; $16.7 \%$ vs $26 \%, \mathrm{p}=0.49)$ were significant predictors of 30 -day mortality.

Conclusion Despite high technical and clinical success, short term mortality is high in this group of patients. GPS, albumin and CRP do not predict outcomes. Further research to optimise patient selection for biliary intervention and strategies to reduce post-procedural cholangitis is warranted.

\section{REFERENCES}

1. Harvey PR, Baldwin S, Mytton J, et al. Higher volume providers are associated with improved outcomes following ERCP for the palliation of malignant biliary obstruction. EClinicalMedicine. 2020 Jan 3;18:100212.

2. Rees J, Mytton J, Evison F, et al. The outcomes of biliary drainage by percutaneous transhepatic cholangiography for the palliation of malignant biliary obstruction in England between 2001 and 2014: a retrospective cohort study. BMJ Open 2020;10:e033576.

\section{PTU-4 PROSPECTIVE VALIDATION OF THE EDINBURGH DYSPHAGIA SCORE IN SOUTH-EAST LONDON}

${ }^{1}$ Miriam Harley*, ${ }^{1}$ Lucia Yin, ${ }^{2}$ Hermon Amanuel, ${ }^{2}$ Sebastian Zeki, ${ }^{3}$ Jale Burch, ${ }^{3}$ Mayur Kumar, ${ }^{4}$ Giles Walker, ${ }^{4}$ David Dewar, ${ }^{1}$ Bu'Hussain Hayee. ${ }^{1}$ King's College Hospital Nhs Foundation Trust, Denmark Hill, London, UK; ${ }^{2}$ Guy's and St Thomas's NHS Foundation Trust, London, UK; ${ }^{3}$ Princess Royal University Hospital, London, UK; ${ }^{4}$ University Hospital Lewisham, London, UK

\subsection{6/gutjnl-2021-BSG.77}

Introduction Although dysphagia should be considered a highrisk symptom for cancer referral pathways, the yield of endoscopy for cancer is currently $<5 \%$. Even prior to the COVID19 pandemic, this was a significant burden for endoscopy units and subjected patients to inappropriate concern at being referred and investigated for suspected cancer. The BSG endorsed use of the Edinburgh Dysphagia Score (cut-off 3.5) to target endoscopy for those at highest risk, ${ }^{1}$ based on previously published results ${ }^{2}$.

Methods Use of the EDS was agreed as a service addition during the COVID-19 recovery period in SE London. All patients referred for dysphagia on the suspected cancer pathway in four acute Trusts were contacted either prior or on arrival in the endoscopy unit to complete the EDS, but the result did not alter the intended pathway (ie. all patients still completed endoscopy). Patients were then followed to final or working diagnosis as determined by the direct care team. As the EDS was primarily designed to prioritise for cancer diagnosis, this was the primary outcome measure. All other diagnoses were recorded and considered 'significant' if felt to be the cause of the patient's presentation. Data on ethnicity was collected to ensure the reliability of the EDS in diverse patient groups found across SE London.

Results 240 patients (117F; mean age $55.7 \pm 14.7 \mathrm{y} ; \mathrm{n}=112$ (46.7\%) non-White British) completed their investigation pathway with $20(8 \%)$ cancers diagnosed. 125 reported EDS $<3.5$ and none had upper GI cancer, while all 20 cancers occurred in the ESD $>3.5$ group $(n=115 ; 17.3 \%$; median score $8(7-9))$. Significant diagnoses were found in 35 patients $(28 \%)$ with EDS $<3.5$ and $48(41.7 \%)$ in the $>3.5$ group.

Conclusion The EDS cut-off of 3.5 had a $100 \%$ negative predictive value in this new prospective cohort and would have resulted in a more-than-doubling of 'hit-rate' for diagnosis of cancer. The rate of significant diagnoses in the $<3.5$ cohort emphasises that investigations are still required, but adoption of the EDS could be used to safely defer or divert patients, avoiding inappropriate use of suspected cancer pathways.

\section{REFERENCE}

1. Br J Surg 2010;97(12):1831-7. www.bsg.org.uk

\section{PTU-5 EFFICACY OF CHOLANGIOSCOPIC DELIVERED ELECTROHYDRAULIC LITHOTRIPSY IN A TERTIARY REFERRAL UNIT}

Andra Caracostea*, Luke Lake, Sreelakshmi Kotha, Ben Warner, Terry Wong, Philip Berry. Guy's And St Thomas' Hospital, London, UK

10.1136/gutjnl-2021-BSG.78 